\title{
The Effect of Low Alcohol Consumption during Pregnancy on the Metabolic Processes of Women and Their Alcohol-Exposed Babies
}

\author{
Anait Marianian*, $\mathrm{PhD}, \mathrm{ScD}$; Marina Darenskaya, $\mathrm{PhD}, \mathrm{ScD}$; \\ Lyudmila Grebenkina PhD, ScD; Natalia Protopopova, PhD, ScD; \\ Lubov Kolesnikova, $\mathrm{PhD}, \mathrm{ScD}$, Member of the RAS \\ Scientific Centre for Family Health and Human Reproduction Problems \\ Irkutsk, the Russian Federation
}

\begin{abstract}
The aim of this study was to examine the effect of low or very low amounts of alcohol consumption on the LPO-AOD systems of pregnant women and their infants after birth, and the effect of that exposure on infant, growth, health, and development.

Methods and Results: A sample of 201 pregnant women (mother-child dyads) was recruited for the study. Pregnant women were categorized into three groups according to the amount of alcohol they consume: 1) non-drinking, 2) very low drinking, and 3) low drinking. Small amounts of alcohol consumption caused dysfunction of the LPO-AOD system and the development of OS in women, and had negative effects on infants.

The biomarkers of potentially harmful LPO, such as thiobarbituric acid reactants (TBARs), were higher in very low and low drinking mothers. The activity of the AOD system was lower among mothers who drank alcohol. Alcohol consumption decreased levels of retinol, SOD activity, GSH, and GR activity. Higher rates of pathological conditions, delayed development, and slower growth were observed among infants who were prenatally exposed to alcohol.

Conclusion: Identification and preventive interventions are needed for pregnant women who use alcohol in any amount. (International Journal of Biomedicine. 2021;11(4):519-525.)

Key Words: lipid peroxidation $\bullet$ antioxidant defense $\bullet$ pregnant women $\bullet$ infant $\bullet$ alcohol

For citation:Marianian A, Darenskaya M, Grebenkina L, Protopopova N, Kolesnikova L. The Effect of Low Alcohol Consumption during Pregnancy on the Metabolic Processes of Women and Their Alcohol-Exposed Babies. International Journal of Biomedicine. 2021;11(4):519-525. doi:10.21103/Article11(4)_OA19
\end{abstract}

\section{Abbreviations}

AOD, antioxidant defense; CDs, conjugated dienes; GPO, glutathione peroxidase; GSH, reduced glutathione; GSSG, oxidized glutathione; GST, glutathione-S-transferase; GR, glutathione reductase; LPO, lipid peroxidation; OS, oxidative stress; SOD, superoxide dismutase; TBARs, thiobarbituric acid reactants.

\section{Introduction}

The period of organogenesis in embryonic development is critical because alcohol exposure during this time has longterm effects. One of the reasons for the teratogenic effect of alcohol is its rapid penetration through the placenta and blood-brain barrier, where alcohol can have effects that are more serious than those of many other substances, including exposure to maternal smoking and illicit drug use. (1-5) $^{-1}$
There is evidence that the fetus is exposed to the same alcohol level as the mother. The effects of alcohol on the fetus are extremely destructive and damaging. The severity of the damage depends on many factors: maternal age, social environment, amount of alcohol consumed, frequency of alcohol consumption, duration of maternal alcohol abuse, and other factors. ${ }^{(6-17)}$

The biotransformation of ethanol is a typical reaction of toxification, which yields metabolites that are more toxic than 
ethanol. ${ }^{(4,17,18)}$ As shown in numerous studies, even small doses of ethanol and its metabolites, especially acetaldehyde, lead to congenital malformations of the child, directly or indirectly, through disruption of maternal biochemical mechanisms, and result in fetal alcohol syndrome..$^{(1,4,8)}$

It is known that an imbalance in the LPO-AOD system leads to the development of OS, which is accompanied by a decrease in the body's resistance to adverse responses to the external and internal environment. ${ }^{(19-26)}$ This imbalance can be assessed by measuring oxidized and reduced forms of glutathione, the activity of enzymes affecting glutathione metabolism (SOD, GR, GPO, and GST), substances that protect from OS ( $\alpha$-tocopherol and retinol), and products that result from oxidation (CDs and TBARS).

Few studies have reported the relationship between alcohol consumption and activities of components of the LPO-AOD system among mothers and infants, and how prenatal alcohol exposure affects infant growth, health, and development by the age of one year.

The aim of this study was to examine the effect of low or very low amounts of alcohol consumption on the LPOAOD systems of pregnant women and their infants after birth, and the effect of that exposure on infant, growth, health, and development.

\section{Materials and Methods}

\section{Study participants}

Pregnant women consecutively enrolled in prenatal care at the Irkutsk Regional Perinatal Center (IRPC) between June 2012 and June 2014 were recruited to participate. Those women who initiated prenatal care at $\leq 10$ weeks of gestation were eligible to participate. Since the study included motherchild dyads, if pregnancy was terminated or did not result in a live birth, the participant was removed from the study.

A total of 204 pregnant women were approached about participating in the study. Two refused to participate, resulting in a sample of 202 pregnant women (mother-child dyads) recruited for the study. All participants were admitted for delivery to IRPC. One delivery resulted in the neonate's death due to severe fetal distress and hypoxia within 24 hours of delivery, resulting in a final sample of 201 mother-child dyads.

\section{Procedures and data collection}

At the first prenatal visit, the women underwent standard medical procedures, including a prenatal care medical exam, were prescribed folic acid, and were provided with information about a healthy lifestyle during pregnancy (risks of alcohol use, tobacco use, and secondhand smoke). All participants completed a face-to-face interview with a study investigator and a brief intervention recommended for alcohol use. ${ }^{(27)}$ At 30-32 weeks of gestation, maternal laboratory tests were completed. At delivery, a neonatologist examined neonates and samples of cord blood were collected. One-to-three days after delivery, mothers completed a face-to-face interview with the study investigator. Follow-up data on infant development were collected from child medical charts.

Collected data included maternal medical history and birth outcomes. Infant medical information noted by a developmental pediatrician at 6 and 12 months was extracted from the hospital's patient records. Characteristics of interest included socio-demographic characteristics, such as maternal age at the time of the birth and marital status; maternal medical history, including number of previous deliveries (parity) and chronic conditions; infectious diseases, such as HIV; pregnancy complications and mode of delivery (vaginal delivery or cesarean section); sex of the neonate, and; the neonate's birth weight, gestational age at birth, and evaluation (APGAR Score) at birth by a neonatologist.

Two face-to-face structured interviews were conducted with each participant by a study investigator. The interviews utilized measures developed by the Prevent FAS Research Group. ${ }^{(28)}$ The first 20- to 30-minute interview was conducted at the time of the first prenatal visit to collect socio-demographic information and to assess women's drug use, alcohol use, smoking, and other risk factors prior to pregnancy. The second interview was conducted on the first to the third day after delivery to collect information about alcohol use and smoking during pregnancy, and took approximately 20 minutes to complete. The postnatal timing of reports was selected due to recent research data suggesting that pregnant women's reports about their consumption during pregnancy are more affected by biases than are their retrospective, after-pregnancy reports about drinking during pregnancy. ${ }^{(29)}$ Several additional measures were implemented to further improve accuracy and elicit truthful self-reports. All interviews were conducted in a clinical setting in private. Participants were alcohol-free when interviewed and were reassured of confidentiality. Questions were worded clearly.

\section{Alcohol exposure}

Participants were asked to provide detailed reports about their alcohol consumption during 40 weeks of pregnancy. Following guidance from Sobell \& Sobell (2003), participants were provided with a calendar and asked to memorize personal events that might be associated with alcohol use, such as holidays and birthdays. Although self-reports about alcohol consumption may be affected by desirability bias, they are considered to be reasonably accurate among volunteers recruited in health care settings when confidentiality is protected. $^{(30-33)}$

The concept of "one drink" as a unit of consumption was not familiar to women in Siberia. Therefore, similar to beverage- and container-specific approaches that have been used in Russia( ${ }^{(8)}$ and other countries, ${ }^{(34,35)}$ a beveragespecific approach was used to determine standardized alcohol content and volume of alcohol consumption. Participants were provided with a card that showed pictures of alcoholic beverages and containers that are common in Russia, and were asked about the type of beverage, type of container, and a number of containers consumed during each month of pregnancy. This information was converted into ethanol volume. The total amounts of alcohol consumed during the first half (1-20 weeks of gestation) and the second half (21-40 weeks) of pregnancy were calculated for each participant and utilized in data analysis. For reporting clarity, these data were then converted to U.S. standard drink units (i.e., 14 grams of pure alcohol). ${ }^{(36)}$ The women were categorized as having very 
low alcohol consumption if they drank less than $750 \mathrm{ml}$ of beer, dry wine, or champagne, and were categorized as having low alcohol consumption if they consumed between $750 \mathrm{ml}$ and $3850 \mathrm{ml}$ of beer, dry wine, or champagne. The control group was women who never consumed alcohol during pregnancy.

\section{Parameters of LPO-AOD system}

Between 30 and 32 weeks gestation, fasting blood was drawn to measure indicators of LPO-AOD system function among pregnant women.

The SOD, GR, GST, and GPO activity was measured in erythrocytes using a commercially available kit. Fluorometry was used to measure GSH and GSSG levels in hemolysate. (37) Retinol and $\alpha$-tocopherol levels were detected in plasma by fluorometry. ${ }^{(38)}$ The concentration of CDs absorbance was detected on plasma heptane extracts at $232 \mathrm{~nm} .{ }^{(39)}$ The coefficient of molar absorption $\left(\mathrm{K}=2.2 \cdot 10^{5} \mathrm{M}^{-1} \mathrm{~S}^{-1}\right)$ for conversation of absorption units of $\mu \mathrm{mol} / \mathrm{L}$ was used. TBARs levels were detected by fluorometry in $\mu \mathrm{mol} / \mathrm{L}$. ${ }^{(40)}$

Umbilical cord blood samples were obtained from $140(69.7 \%)$ of 201 infants; $66(70.1 \%)$ of 93 infants in Group $1 ; 53(70.1 \%)$ of 75 infants in Group 2, and; 21(63.6\%) of 33 infants in Group 3. We examined the same markers of the LPO-AOD system in infants as we did in their mothers.

\section{Child developmental evaluation}

Child development data, including infant height, weight, head and chest circumferences, congenital malformations, rickets, and developmental milestones, were extracted from the children's medical charts. Following routine medical procedures and standard protocols, neonates were evaluated at birth $(n=201)$ by their clinic's neonatologists. At 6 and 12 months of age, infants ( $n=201$ ) were evaluated by a pediatrician and a pediatric neurologist at the infant's local pediatric clinic. Anemia was diagnosed based on blood hemoglobin $(\leq 12 \mathrm{~g} /$ $\mathrm{dL}$ for newborns; $<10 \mathrm{~g} / \mathrm{dL}$ for infants aged between 6 and 12 months). Rickets was diagnosed by clinical evaluation by a pediatrician. Congenital malformations included congenital heart disease, retroperitoneal tumor, adrenal gland tumor, malformations, fetal alcohol syndrome spectrum disorders, and cerebral palsy. Pediatric neurologists evaluated all children in the first 2-4 days of life and at 6 and 12 months to evaluate psychomotor development according to developmental milestones.

Statistical processing was carried out using the STATISTICA Version 10 (StatSoft, USA). The normality of distribution of continuous variables was tested by ShapiroWilk test. For descriptive analysis, results are presented as mean \pm standard deviation (SD), median (Me) (interquartile range [IQR]). Multiple comparisons were performed with one-way ANOVA and Tukey's HSD Post-hoc Test. KruskalWallis test was used to compare means of 3 groups of variables not normally distributed. Categorical variables were analyzed using the chi-square test with the Yates' correction. A value of $P<0.05$ was considered significant.

The study was carried out in compliance with Ethical Principles for Medical Research Involving Human Subjects, Adopted by the 18th WMA General Assembly, Helsinki, Finland, June 1964, and amended by the 64th WMA General Assembly, Fortaleza, Brazil, October 2013. The study was approved by the Ethics Committee of the Scientific Center for Family Health and Human Reproduction Problems. Written informed consent was obtained from each patient.

\section{Results}

\section{Clinical characteristics of pregnant women and infants at birth}

We analyzed the clinical characteristics of the surveyed women and their newborns (Table 1). A total of 201 pregnant women aged 15 to $42(29.1 \pm 6.0)$ enrolled in the study at their initiation of prenatal care at 7-to-10 weeks $(7.1 \pm 0.5)$ of gestation. There were no differences in age, marital status, or other socio-demographic characteristics between women who reported consuming alcohol and those who reported no alcohol use during pregnancy. Among participants, 69.2\% were married and $41.8 \%$ had a higher education than a school diploma or secondary education. A total of $37.3 \%$ and $34.8 \%$ reported smoking prior to pregnancy and during pregnancy. None were positive for HIV.

\section{Table 1.}

Characteristics of the surveyed pregnant women

\begin{tabular}{|l|c|c|c|c|}
\hline \multicolumn{1}{|c|}{ Variable } & $\begin{array}{c}\text { Group 1 } \\
(\mathrm{n}=93)\end{array}$ & $\begin{array}{c}\text { Group 2 } \\
(\mathrm{n}=75)\end{array}$ & $\begin{array}{c}\text { Group 3 } \\
(\mathrm{n}=33)\end{array}$ & \multicolumn{1}{|c|}{ Statistics } \\
\hline Age, yrs & $29.7 \pm 0.6$ & $28.3 \pm 0.7$ & $29.0 \pm 1.0$ & $\begin{array}{l}\mathrm{P}=0.0000 \\
\mathrm{P}_{1-2}=0.0000 \\
\mathrm{P}_{1-3}=0.0000 \\
\mathrm{P}_{2-3}=0.0000\end{array}$ \\
\hline Weight, kg & $68.2 \pm 1.2$ & $69.1 \pm 0.3$ & $68.8 \pm 0.4$ & $\begin{array}{l}\mathrm{P}^{2}=0.0000 \\
\mathrm{P}_{1-2}=0.0000 \\
\mathrm{P}_{1-3}=0.0018 \\
\mathrm{P}_{2-3}=0.2144\end{array}$ \\
\hline Height, cm & $166.3 \pm 0.7$ & $166.1 \pm 0.3$ & $166.7 \pm 0.6$ & $\begin{array}{l}\mathrm{P}=0.0000 \\
\mathrm{P}_{1-2}=0.0610 \\
\mathrm{P}_{1-3}=0.0017 \\
\mathrm{P}_{2-3}=0.0000\end{array}$ \\
\hline
\end{tabular}

In this sample $(n=201), 93$ participants reported no alcohol consumption during pregnancy (Group 1), and 108 reported consuming alcohol in some amount. Based on the total amount of alcohol reported during pregnancy, participants were categorized into three groups: no alcohol use during pregnancy (Group 1, $\mathrm{n}=93$ ), very low ( $\leq 2$ drinks) alcohol use (Group 2, $\mathrm{n}=75$ ), and low ( $>2$ drinks) alcohol use (Group 3, $\mathrm{n}=33$ ). The average amounts of alcohol consumed were $1.28 \pm 0.48$ drinks for Group 2 and $5.91 \pm 2.67$ drinks for Group 3.

With regard to tobacco use, $15.1 \%$ of Group 1, $42.7 \%$ of Group 2, and $72.7 \%$ of Group 3 women reported smoking daily or occasionally, less than 10 and not more than 20 cigarettes a day, during pregnancy. Women with higher alcohol consumption during pregnancy smoked more often than did those with lower alcohol consumption during pregnancy (Group 1 vs. Group 2, $P=0.005$; Group 1 vs. Group $3, P=0.001$; Group 2 vs. Group 3. $P=0.04$ ).

There were no significant differences between the heights, weights, and head and chest circumferences of the infants in any of the three groups (Table 2). 
Table 2.

Characteristics of infants who were prenatally exposed to alcohol and infants with no alcohol exposure.

\begin{tabular}{|c|c|c|c|c|}
\hline \multirow[t]{2}{*}{ Variable } & $\begin{array}{l}\text { Group } 1 \\
(\mathrm{n}=93)\end{array}$ & $\underset{(n=75)}{\text { Group } 2}$ & $\begin{array}{c}\text { Group } 3 \\
(\mathrm{n}=33)\end{array}$ & \multirow[t]{2}{*}{ Statistics } \\
\hline & \multicolumn{3}{|c|}{$\mathrm{M} \pm \mathrm{SD} ; \mathrm{Me}(\mathrm{IQR})$} & \\
\hline Height, cm & $50.9 \pm 2.9$ & $50.1 \pm 4.3$ & $49.7 \pm 4.4$ & $\begin{array}{l}\mathrm{P}=0.1937 \\
\mathrm{P}_{1-2}=0.3532 \\
\mathrm{P}^{1-3}=0.2541 \\
\mathrm{P}_{2--3}=0.8652\end{array}$ \\
\hline Weight, kg & $3.3(2.9-3.7)$ & $3.2(2.8-3.7)$ & $3.04(2.6-3.6)$ & $\mathrm{P}>0.05$ \\
\hline $\begin{array}{l}\text { Head } \\
\text { circumference, } \\
\mathrm{cm}\end{array}$ & $33.9 \pm 1.5$ & $33.1 \pm 3.7$ & $33.6 \pm 2.2$ & $\begin{array}{l}\mathrm{P}=0.1494 \\
\mathrm{P}_{1-2}=0.1259 \\
\mathrm{P}_{1-3}=0.8405 \\
\mathrm{P}_{2--3}=0.6357\end{array}$ \\
\hline $\begin{array}{l}\text { Chest } \\
\text { circumference, } \\
\mathrm{cm}\end{array}$ & $32.7 \pm 2.6$ & $31.7 \pm 3.6$ & $31.9 \pm 3.1$ & $\begin{array}{l}\mathrm{P}=0.0973 \\
\mathrm{P}_{1-2}=0.0952 \\
\mathrm{P}_{1-3}=0.4090 \\
\mathrm{P}_{2-3}=0.9484\end{array}$ \\
\hline
\end{tabular}

\section{Development of infants at 6 and 12 months of age}

Congenital malformations were significantly more common in children with low prenatal alcohol exposure at both ages, compared with the children with very low exposure (Table 3 ). Rickets was also significantly more common at 12 months in Group 3 children than in Group 2 children. Hypoxicischemic central nervous system damage in newborns was noted in $2.2 \%$ of infants from Group 1, $8 \%$ of infants from Group 2, and 24.4\% of infants from Group 3.

Table 3.

Congenital malformations and other pathological conditions of infants at 6 and 12 months of age

\begin{tabular}{|c|c|c|c|c|c|}
\hline & Variable & $\begin{array}{c}\text { Group } 1 \\
(\mathrm{n}=93) \\
\mathrm{n}(\%)\end{array}$ & $\begin{array}{c}\text { Group } 2 \\
(\mathrm{n}=75) \\
\mathrm{n}(\%)\end{array}$ & $\begin{array}{c}\text { Group } 3 \\
(n=33) \\
n(\%)\end{array}$ & Statistics \\
\hline \multirow{3}{*}{$\begin{array}{l}\stackrel{y}{D} \\
\stackrel{\Xi}{0} \\
\vdots \\
0\end{array}$} & $\begin{array}{l}\text { Congenital } \\
\text { malformations }\end{array}$ & $4(4.3)$ & $7(9.3)$ & $10(30.3)$ & $\mathrm{P}=0.0001$ \\
\hline & Rickets & $18(19.4)$ & $16(21.3)$ & $7(21.2)$ & $\mathrm{P}=0.9436$ \\
\hline & Anemia & $22(23.7)$ & $18(24.0)$ & $8(24.2)$ & $\mathrm{P}=0.9970$ \\
\hline \multirow{3}{*}{$\begin{array}{l}\stackrel{y}{E} \\
\stackrel{\Xi}{0} \\
\Xi \\
\simeq\end{array}$} & $\begin{array}{l}\text { Congenital } \\
\text { malformations }\end{array}$ & $4(4.3)$ & $7(9.3)$ & $10(30.3)$ & $\mathrm{P}=0.0001$ \\
\hline & Rickets & $10(10.8)$ & $8(10.7)$ & $7(21.2)$ & $\mathrm{P}=0.2477$ \\
\hline & Anemia & $12(12.9)$ & $14(18.7)$ & $8(24.2)$ & $\mathrm{P}=0.2881$ \\
\hline
\end{tabular}

Children in Groups 2 and 3 had significantly lower weights, heights, and head circumferences at 6 and 12 months of age, and significantly larger fontanelles at 12 months of age than did children in Group 1 (Table 4). Infants with very low exposure had significantly lower heights and weights than did infants with no exposure at 6 and 12 months.

In analyzing the physical and psychomotor development of children 6 and 12 months of age, those with low prenatal alcohol exposure were more likely to have psychomotor development delayed for their age. Thus, they had significantly more motor development delay at 6 and 12 months than did children with no exposure (Group 1 vs. Group3 at 6 months. $P=0.023$; Group 1 vs. Group 3 at 12 months, $P=0.015$ ).

Table 4.

Anthropometric data in the infants at the first year of life

\begin{tabular}{|c|c|c|c|c|c|}
\hline & \multirow[t]{2}{*}{ Variable } & $\underset{(\mathrm{n}=93)}{\operatorname{Group}} 1$ & $\begin{array}{c}\text { Group } 2 \\
(\mathrm{n}=75)\end{array}$ & $\begin{array}{c}\text { Group } 3 \\
(\mathrm{n}=33)\end{array}$ & \multirow[t]{2}{*}{ Statistics } \\
\hline & & \multicolumn{3}{|c|}{$\mathrm{M} \pm \mathrm{SD} ; \mathrm{Me}(\mathrm{IQR})$} & \\
\hline \multirow{4}{*}{ 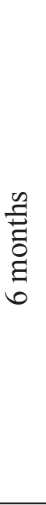 } & Weight, kg & $7.7 \pm 0.5$ & $6.9 \pm 0.5$ & $6.8 \pm 0.6$ & $\begin{array}{l}\mathrm{P}=0.0000 \\
\mathrm{P}_{1-2}=0.0000 \\
\mathrm{P}_{1-2}=0.0000 \\
\mathrm{P}_{2-3}=0.6251\end{array}$ \\
\hline & Height, cm & $70.2 \pm 1.1$ & $67.8 \pm 1.1$ & $67.6 \pm 1.6$ & $\begin{array}{l}\mathrm{P}=0.0000 \\
\mathrm{P}_{1-2}=0.0000 \\
\mathrm{P}_{1-3}=0.0000 \\
\mathrm{P}_{2-3}=0.7027\end{array}$ \\
\hline & $\begin{array}{l}\text { Head } \\
\text { circumference, } \\
\text { cm }\end{array}$ & $43.7 \pm 1.4$ & $45.9 \pm 0.42$ & $40.7 \pm 0.9$ & $\begin{array}{l}\mathrm{P}=0.0000 \\
\mathrm{P}_{1-2}=0.0000 \\
\mathrm{P}_{1-3}=0.0000 \\
\mathrm{P}_{2-3}=0.0000\end{array}$ \\
\hline & $\begin{array}{l}\text { Large fontanelle, } \\
\mathrm{cm}\end{array}$ & $1.5(1.5-1.5)$ & $1.5(1.5-1.5)$ & $1.5(1.5-1.5)$ & $\mathrm{NaN}$ \\
\hline \multirow{4}{*}{ 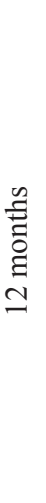 } & Weight, $\mathrm{kg}$ & $12.3 \pm 0.9$ & $10.3 \pm 1.2$ & $10.0 \pm 1.0$ & $\begin{array}{l}\mathrm{P}=0.0000 \\
\mathrm{P}_{1-2}=0.0000 \\
\mathrm{P}_{1-3}=0.0000 \\
\mathrm{P}_{2-3}=0.3510\end{array}$ \\
\hline & Height, cm & $81.2 \pm 2.0$ & $78.1 \pm 1.8$ & $77.6 \pm 2.0$ & $\begin{array}{l}\mathrm{P}=0.0000 \\
\mathrm{P}_{1-2}=0.0000 \\
\mathrm{P}^{1-3}=0.0000 \\
\mathrm{P}_{2-3}=0.4301\end{array}$ \\
\hline & $\begin{array}{l}\text { Head } \\
\text { circumference, } \\
\text { cm }\end{array}$ & $48.8 \pm 0.9$ & $51.4 \pm 0.5$ & $44.5 \pm 1.3$ & $\begin{array}{l}\mathrm{P}=0.0000 \\
\mathrm{P}_{1-2}=0.0000 \\
\mathrm{P}_{1-3}=0.0000 \\
\mathrm{P}_{2-3}=0.0000\end{array}$ \\
\hline & $\begin{array}{l}\text { Large fontanelle, } \\
\mathrm{cm}\end{array}$ & $1.0(0.5-1.0)$ & $1.0(0.5-1.0)$ & $1.0(0.5-1.0)$ & $\mathrm{NaN}$ \\
\hline
\end{tabular}

\section{Characteristics of LPO-AOD process}

The level of CDs was significantly lower in women of Group 2 than in women of Group $1(P=0.003)$. The concentrations of TBARs in Groups 2 and 3 were significantly higher than those in Group 1 (Table 5).

There were no statistically significant differences in $\alpha$-tocopherol levels between the groups. However, retinol concentrations were significantly lower in both drinking groups than in the non-drinking group. Dysregulation in the glutathione system was indicated by significantly lower GSH values and GR activity, and higher GSSG and GST levels, among women of Group 1 than in women of Group 3.

Table 6 indicates the levels of markers related to the LPO-AOD system in infants who were prenatally exposed to alcohol and infants with no alcohol exposure. CDs were statistically significantly higher in infants from Groups 2 and 3 than in infants from Group 1. In the AOD system, the values of $\alpha$-tocopherol and SOD activity were significantly lower in Groups 2 and 3 than in Group 1. In terms of changes in the glutathione system, there were significantly lower levels of 
GSH in Group 2 and GR in Groups 2 and 3 than in Group 1. Lower GR in Groups 2 and 3 was accompanied by a decrease in the activity of the other glutathione-dependent enzymes, GST and GP.

Table 5.

LPO-AOD system components in the pregnant women of the study groups

\begin{tabular}{|c|c|c|c|c|}
\hline \multirow[t]{2}{*}{ Variable } & $\begin{array}{c}\text { Group } 1 \\
(\mathrm{n}=93)\end{array}$ & $\begin{array}{c}\text { Group } 2 \\
(\mathrm{n}=75)\end{array}$ & $\begin{array}{c}\text { Group } 3 \\
(\mathrm{n}=33)\end{array}$ & \multirow[t]{2}{*}{ Statistics } \\
\hline & \multicolumn{3}{|c|}{$\mathrm{M} \pm \mathrm{SD} ; \mathrm{Me}(\mathrm{IQR})$} & \\
\hline $\begin{array}{l}\text { CDs, } \\
\mu \mathrm{mol} / \mathrm{L}\end{array}$ & $\mid \begin{array}{c}2.12 \\
(1.61-2.68)\end{array}$ & $\begin{array}{c}1.19 \\
(1.26-2.50)\end{array}$ & $\begin{array}{c}1.84 \\
(1.50-2.40)\end{array}$ & $\begin{array}{l}\mathrm{P}_{1-2}=0.003 \\
\mathrm{P}_{1-3}=0.088 \\
\mathrm{P}_{2-3}=0.989\end{array}$ \\
\hline $\begin{array}{l}\text { TBARs, } \\
\mu \mathrm{mol} / \mathrm{L}\end{array}$ & $\mid \begin{array}{c}0.83 \\
(0.61-1.12)\end{array}$ & $\begin{array}{c}1.19 \\
(0.7-1.57)\end{array}$ & $\begin{array}{c}1.18 \\
(0.87-1.54)\end{array}$ & $\begin{array}{l}\mathrm{P}_{1-2}<0.001 \\
\mathrm{P}_{1-3}<0.001 \\
\mathrm{P}_{2-3}=0.951\end{array}$ \\
\hline $\begin{array}{l}\alpha \text {-tocopherol, } \\
\mu \mathrm{mol} / \mathrm{L}\end{array}$ & $\begin{array}{c}6.49 \\
(5.48-7.77)\end{array}$ & $\begin{array}{c}5.46 \\
(4.10-8.07)\end{array}$ & $\begin{array}{c}6.33 \\
(4.77-9.88) \\
\end{array}$ & $\begin{array}{l}\mathrm{P}_{1-2}=0.243 \\
\mathrm{P}_{1-3}=0.167 \\
\mathrm{P}_{2-3}=0.053\end{array}$ \\
\hline $\begin{array}{l}\text { Retinol, } \\
\mu \mathrm{mol} / \mathrm{L}\end{array}$ & $\begin{array}{c}0.75 \\
(0.59-0.92)\end{array}$ & $\begin{array}{c}0.58 \\
(0.431-0.70)\end{array}$ & $\begin{array}{c}0.57 \\
(0.48-0.67) \\
\end{array}$ & $\begin{array}{l}\mathrm{P}_{1-2}<0.001 \\
\mathrm{P}_{1-3}<0.001 \\
\mathrm{P}_{2-3}=0.769\end{array}$ \\
\hline $\begin{array}{l}\text { SOD, } \\
\text { U/mgHb }\end{array}$ & $1.69 \pm 0.15$ & $1.63 \pm 0.19$ & $1.64 \pm 0.19$ & $\begin{array}{l}\mathrm{P}=0.0658 \\
\mathrm{P}_{1-2}=0.0670 \\
\mathrm{P}_{1-3}=0.3275 \\
\mathrm{P}_{2-3}=0.9585\end{array}$ \\
\hline $\begin{array}{l}\text { GSH, } \\
\mathrm{mmol} / \mathrm{L}\end{array}$ & $\begin{array}{c}2.11 \\
(1.85-2.41)\end{array}$ & $\begin{array}{c}1.96 \\
(1.81-2.24)\end{array}$ & $\begin{array}{c}2.09 \\
(1.72-2.39)\end{array}$ & $\begin{array}{l}\mathrm{P}_{1-2}=0.014 \\
\mathrm{P}_{1-3}=0.412 \\
\mathrm{P}_{2-3}=0.334\end{array}$ \\
\hline $\begin{array}{l}\text { GSSG, } \\
\mathrm{mmol} / \mathrm{L}\end{array}$ & $\begin{array}{c}1.81 \\
(1.5-2.13)\end{array}$ & $\begin{array}{c}1.92 \\
(1.75-2.26)\end{array}$ & $\begin{array}{c}1.97 \\
(1.78-2.25)\end{array}$ & $\begin{array}{l}\mathrm{P}_{1-2}=0.023 \\
\mathrm{P}_{1-3}=0.025 \\
\mathrm{P}_{2-3}=0.717\end{array}$ \\
\hline $\begin{array}{l}\mathrm{GR}, \\
\mu \mathrm{mol} / \mathrm{min} / \mathrm{L}\end{array}$ & $\begin{array}{c}951 \\
(752-1168)\end{array}$ & $\begin{array}{c}764 \\
(459-965)\end{array}$ & $\begin{array}{c}855 \\
(541-1097) \\
\end{array}$ & $\begin{array}{l}\mathrm{P}_{1-2}<0.001 \\
\mathrm{P}_{1-3}=0.068 \\
\mathrm{P}_{2-3}=0.434\end{array}$ \\
\hline $\begin{array}{l}\mathrm{GST}, \\
\mu \mathrm{mol} / \mathrm{min} / \mathrm{L}\end{array}$ & $\begin{array}{c}809 \\
(678-951)\end{array}$ & $\begin{array}{c}966 \\
(852-1450)\end{array}$ & $\begin{array}{c}852 \\
(457-1423) \\
\end{array}$ & $\begin{array}{l}\mathrm{P}_{1-2}<0.001 \\
\mathrm{P}_{1-3}=0.068 \\
\mathrm{P}_{2-3}=0.434\end{array}$ \\
\hline $\begin{array}{l}\mathrm{GPO}, \\
\mu \mathrm{mol} / \mathrm{min} / \mathrm{L}\end{array}$ & $\begin{array}{c}279 \\
(216-316)\end{array}$ & $\begin{array}{c}256 \\
(197-308)\end{array}$ & $\begin{array}{c}275 \\
(206-321)\end{array}$ & $\begin{array}{l}\mathrm{P}_{1-2}=0.260 \\
\mathrm{P}_{1-3}=0.895 \\
\mathrm{P}_{2-3}=0.364\end{array}$ \\
\hline
\end{tabular}

\section{Table 6.}

LPO-AOD components in infants who were prenatally exposed to alcohol and infants with no alcohol exposure

\begin{tabular}{|l|c|c|c|c|}
\hline \multirow{2}{*}{ Variable } & $\begin{array}{c}\text { Group 1 } \\
(\mathrm{n}=66)\end{array}$ & $\begin{array}{c}\text { Group 2 } \\
(\mathrm{n}=53)\end{array}$ & $\begin{array}{c}\text { Group 3 } \\
(\mathrm{n}=21)\end{array}$ & Statistics \\
\cline { 2 - 4 } & \multicolumn{2}{|c|}{$\mathrm{M} \pm \mathrm{SD}$; Me (IQR) } & \\
\hline $\begin{array}{l}\mathrm{CDs}, \\
\mu \mathrm{mol} / \mathrm{L}\end{array}$ & $\begin{array}{c}1.34 \\
(1.12-1.67)\end{array}$ & $\begin{array}{c}1.73 \\
(0.98-2.13)\end{array}$ & $\begin{array}{c}1.68 \\
(1.52-2.13)\end{array}$ & $\begin{array}{l}\mathrm{P}_{1-2}=0.006 \\
\mathrm{P}^{1-3}=0.001 \\
\mathrm{P}_{2-3}=0.396\end{array}$ \\
\hline $\begin{array}{l}\text { TBARs, } \\
\mu \mathrm{mol} / \mathrm{L}\end{array}$ & $\begin{array}{c}1.32 \\
(1.06-1.61)\end{array}$ & $\begin{array}{c}1.35 \\
(1.16-1.64)\end{array}$ & $\begin{array}{c}1.31 \\
(0.90-1.44)\end{array}$ & $\begin{array}{l}\mathrm{P}_{1-2}^{1-2}=0.976 \\
\mathrm{P}_{1-3}^{1-3}=0.764 \\
\mathrm{P}_{2-3}=0.771\end{array}$ \\
\hline $\begin{array}{l}\alpha-\text { tocopherol, } \\
\mu \mathrm{mol} / \mathrm{L}\end{array}$ & $\begin{array}{c}6.98 \\
(5.84-8.17)\end{array}$ & $\begin{array}{c}5.88 \\
(5.22-7.05)\end{array}$ & $\begin{array}{c}6.08 \\
(5.21-7.15)\end{array}$ & $\begin{array}{l}\mathrm{P}_{1-2}^{1-2}=0.006 \\
\mathrm{P}_{1-3}^{1-3}=0.029 \\
\mathrm{P}_{2-3}=0.823\end{array}$ \\
\hline $\begin{array}{l}\text { Retinol, } \\
\mu \mathrm{mol} / \mathrm{L}\end{array}$ & $0.41 \pm 0.10$ & $0.43 \pm 0.11$ & $0.42 \pm 0.10$ & $\begin{array}{l}\mathrm{P}_{1-2}^{1-2}=0.385 \\
\mathrm{P}_{1-3}=0.876 \\
\mathrm{P}_{2-3}=0.460\end{array}$ \\
\hline
\end{tabular}

Table 6 (continued).

$L P O-A O D$ components in infants who were prenatally exposed to alcohol and infants with no alcohol exposure

\begin{tabular}{|c|c|c|c|c|}
\hline \multirow[t]{2}{*}{ Variable } & $\begin{array}{c}\text { Group } 1 \\
(\mathrm{n}=66)\end{array}$ & $\begin{array}{c}\text { Group } 2 \\
(\mathrm{n}=53)\end{array}$ & $\begin{array}{c}\text { Group } 3 \\
(\mathrm{n}=21)\end{array}$ & \multirow[t]{2}{*}{ Statistics } \\
\hline & \multicolumn{3}{|c|}{$\mathrm{M} \pm \mathrm{SD} ; \mathrm{Me}(\mathrm{IQR})$} & \\
\hline $\begin{array}{l}\text { SOD, } \\
\text { U/mg Hb }\end{array}$ & $1.74 \pm 0.08$ & $1.64 \pm 0.14$ & $1.69 \pm 0.13$ & $\begin{array}{l}\mathrm{P}_{1-2}<0.010 \\
\mathrm{P}_{1-3}=0.030 \\
\mathrm{P}_{2--3}=0.109\end{array}$ \\
\hline $\begin{array}{l}\mathrm{GSH}, \\
\mathrm{mmol} / \mathrm{L}\end{array}$ & $2.26 \pm 0.40$ & $2.04 \pm 0.28$ & $2.15 \pm 0.29$ & $\begin{array}{l}\mathrm{P}_{1-2}=0.014 \\
\mathrm{P}^{1-3}=0.223 \\
\mathrm{P}_{2--3}=0.139\end{array}$ \\
\hline $\begin{array}{l}\text { GSSG, } \\
\mathrm{mmol} / \mathrm{L}\end{array}$ & $\begin{array}{c}1.97 \\
(1.66-2.20)\end{array}$ & $\begin{array}{c}1.96 \\
(1.78-2.29)\end{array}$ & $\begin{array}{c}1.96 \\
(1.59-2.06)\end{array}$ & $\begin{array}{l}\mathrm{P}_{1-2}=0.781 \\
\mathrm{P}^{1-3}=0.197 \\
\mathrm{P}_{2--3}=0.151\end{array}$ \\
\hline $\begin{array}{l}\mathrm{GR}, \\
\mu \mathrm{mol} / \mathrm{min} / \mathrm{L}\end{array}$ & $\begin{array}{c}857.5 \\
(731-985)\end{array}$ & $\begin{array}{c}652 \\
(456-851)\end{array}$ & $\begin{array}{c}699 \\
(456-1085)\end{array}$ & $\begin{array}{l}\mathrm{P}_{1-2}<0.001 \\
\mathrm{P}^{1-3}<0.001 \\
\mathrm{P}_{2-3}=0.277\end{array}$ \\
\hline $\begin{array}{l}\mathrm{GST}, \\
\mu \mathrm{mol} / \mathrm{min} / \mathrm{L}\end{array}$ & $\begin{array}{c}1244 \\
(1056-1478)\end{array}$ & $\begin{array}{c}995 \\
(852-1431)\end{array}$ & $\begin{array}{c}1133 \\
(813-1455)\end{array}$ & $\begin{array}{l}\mathrm{P}_{1-2}=0.012 \\
\mathrm{P}^{1-3}=0.041 \\
\mathrm{P}_{2-3}=0.961\end{array}$ \\
\hline $\begin{array}{l}\mathrm{GPO}, \\
\mu \mathrm{mol} / \mathrm{min} / \mathrm{L}\end{array}$ & $\begin{array}{c}280 \\
(231-318)\end{array}$ & $\begin{array}{c}231 \\
(199-291)\end{array}$ & $\begin{array}{c}245 \\
(233-316)\end{array}$ & $\begin{array}{l}\mathrm{P}_{1-2}=0.002 \\
\mathrm{P}^{1-3}=0.031 \\
\mathrm{P}_{2--3}=0.396\end{array}$ \\
\hline
\end{tabular}

\section{Discussion}

Our findings indicate that even a small amount of alcohol consumed during pregnancy can cause serious metabolic changes in mothers and their newborn babies. In particular, alcohol exposure leads to an imbalance of redox exchange and dysfunction of the LPO-AOD system. The concentrations of TBARs were $60.4 \%$ higher in the very low and low drinking groups than in the control group of non-drinkers. The increase of TBARs indicates the decline of AOD that can be described as the OS development. ${ }^{(22)}$

Retinol concentration decreased in both drinking groups, compared with the control group. This reduction may result in a corresponding reduction in the antioxidant effect of retinol. The role of retinol as a prohormone may also be affected by oxidation, prompting its development into retinoic acid, a true hormone, which is involved in the regulation of gene expression. ${ }^{(21,41)}$ Further, retinoic acid has morphogenetic action, and its deficiency can lead to fetal malformations.

SOD activity was significantly lower in very low drinkers than in non-drinkers. Even a small reduction in SOD activity is an important signal of the metabolic shift in the direction of prevailing pro-oxidant processes, because of the high content of the enzyme in the red blood cell. ${ }^{(21)}$ Women who drank very low amounts of alcohol had lower GSH and GR values, and higher GSSG and GST values, than did those who drank no alcohol. The reduced form of glutathione participates in neutralizing oxidants and in transporting substances across membranes, and has an antitoxic effect. ${ }^{(24,42-44)}$ The decreased GSH and GR levels in women who consumed alcoholic beverages had a negative effect on their health. ${ }^{(45)}$ Reduced activity of GR in women who consumed even very low doses of alcohol, in turn, indicates the protective function of the enzyme, conversion of GSSG to GSH. 
Infants prenatally exposed to small and moderate amounts of alcohol had higher rates of pathological conditions, smaller heights and weights, larger fontanelles, and smaller head circumferences at birth and 6 and 12 months of age, as well as delayed psychomotor development than those with no alcohol exposure. In terms of the LPO-AOD system of newborns, changes similar to those that occurred in their mothers were found, especially for SOD, GSH, and GR. The decrease in GSH concentration and activity of the enzymes involved in its metabolism, which were found in infants of Group 2, has a negative effect on the balance in the LPO-AOD system. It is reported that $\alpha$-tocopherol activated $\gamma$-glutamyl cysteine synthetase, which leads to regulation of glutathione biosynthesis. In this case, $\alpha$-tocopherol has an indirect effect on the AOD system. ${ }^{(1)}$

The assessments of the LPO-AOD system for women who consumed alcoholic beverages in the prenatal period indicate increased OS, even when consuming low doses. Thus, the results suggest that even a small amount of alcohol drunk by women during pregnancy can cause serious metabolic changes in the newborn body, leading to an imbalance of redox exchange and dysfunction of the LPO-AOD system.

\section{Conclusion}

The use of even small doses of alcohol during pregnancy can cause dysfunction of the LPO-AOD system and the development of OS and can have negative effects on infant growth, health, and development. Specialists and researchers in the medical and social fields must address the prevention of any amount of alcohol use in pregnant women.

\section{Acknowledgments}

This article contains material that has been discussed at the VIII International Research and Practical Conference «FUNDAMENTAL AND APPLIED ASPECTS OF REPRODUCTION» (December 2021, Irkutsk, Russia). The author thanks all researchers who participated in the oral discussion.

\section{Conflict of Interests} interest.

The authors declare that they have no conflicts of

\section{References}

1. Maier SE, West JR. Drinking patterns and alcohol-related birth defects. Alcohol Res Health. 2001;25(3):168-74.

2. Randall CL. Alcohol and pregnancy: highlights from three decades of research. J Stud Alcohol. 2001 Sep;62(5):554-61. doi: 10.15288/jsa.2001.62.554.

3. Riley EP, McGee CL. Fetal alcohol spectrum disorders: An overview with emphasis on changes in brain and behavior. Exp Biol Med (Maywood). 2005;230(6):357-365. doi: 10.1177/15353702-0323006-03.

4. Skagerstróm J, Chang G, Nilsen P. Predictors of drinking during pregnancy: a systematic review. J Womens Health
(Larchmt). 2011 Jun;20(6):901-13. doi: 10.1089/jwh.2010.2216. 5. Thackray H, Tifft C. Fetal alcohol syndrome. Pediatr Rev. 2001;22(2):47-55. doi: 10.1542/pir.22-2-47.

6. Abel EL, Dintcheff BA. Factors affecting the outcome of maternal alcohol exposure: II. Maternal age. Neurobehav Toxicol Teratol. 1985 May-Jun;7(3):263-6.

7. Abel EL, Hannigan JH. Maternal risk factors in fetal alcohol syndrome: provocative and permissive influences. Neurotoxicol Teratol. 1995 Jul-Aug;17(4):445-62. doi: 10.1016/0892-0362(95)98055-6. Erratum in: Neurotoxicol Teratol 1995 Nov-Dec;17(6):689.

8. Balachova T, Bonner B, Chaffin M, Bard D, Isurina G, Tsvetkova L et al. Women's alcohol consumption and risk for alcohol-exposed pregnancies in russia. Addiction. 2012;107(1):109-117. doi: 10.1111/j.1360-0443.2011.03569.x 9. Darenskaya MA, Kolesnikov SI, Rychkova LR, Grebenkina LA, Kolesnikova LI. Oxidative stress and antioxidant defense parameters in different diseases: Ethnic aspects. Free Radical Biology and Medicine. 2018;120(1):60. 10. Gilliam D, Irtenkauf K. Maternal genetic effects on ethanol teratogenesis and dominance of relative embryonic resistance to malformations. Alcohol Clin Exp Res. 1990;14(4):539-545. doi: 10.1111/j.1530-0277.1990.tb01196.x.

11. Jacobson JL, Jacobson SW, Sokol RJ, Martier SS, Ager JW, Shankaran S. Effects of alcohol use, smoking, and illicit drug use on fetal growth in black infants. J Pediatr. 1994 May; 124(5 Pt 1):757-64. doi: 10.1016/s0022-3476(05)81371-X.

12. Kosyh E, Balachova T, Bonner B, Volkova E. Alcohol consumption by pregnant women in the Nizhny Novgorod region, Russia. Alcohol Clin Exp Res. 2010; 34(3):111.

13. Marianian AYu, Molchanova EV. Social and Economic Effect of Comprehensive Prevention of Fetal Alcohol Syndrome and Fetal Alcohol Spectrum Disorders in Children: A Review. Journal of Pharmaceutical Research International. 2020; 32(23): 115-123. doi: 10.9734/jpri/2020/v32i2330798.

14. McCarver DG, Thomasson HR, Martier SS, Sokol RJ, Li T. Alcohol dehydrogenase- $2 * 3$ allele protects against alcoholrelated birth defects among african americans. J Pharmacol Exp Ther. 1997;283(3):1095-1101.

15. West JR. Fetal alcohol-induced brain damage and the problem of determining temporal vulnerability: A review. Alcohol Drug Res. 1987;7(5-6):423-441.

16. West JR, Blake CA. Fetal alcohol syndrome: an assessment of the field. Exp Biol Med (Maywood). 2005 Jun;230(6):3546. doi: 10.1177/15353702-0323006-02.

17. Yaltonskaya A, Yaltonsky V, Kolpakov Y, Abrosimov I, Tanner, V, Pervakov, K, Rehm J, Popova S. The alcohol consumption during pregnancy and fetal alcohol spectrum disorders in russia: Systematic literature reviews of scientific. Monthly reviewed scientific and practical journal Russian an Academy of Medical Sciences International Society of Addiction Journal Editors. 2014;6:80-90.

18. Rychkova L, Marianian A, Darenskaya M, Grebenkina L, Barbara B, Balachova $\mathrm{T}$ et al. Lipid peroxidation processes in newborns born from mothers who consumed alcohol during pregnancy Archives of Disease in Childhood. 2017;102(2):A90-A91. doi: 10.1136/ archdischild-2017-313273.235.

*Corresponding author: Anait Marianian, PhD, ScD. Scientific Centre for Family Health and Human Reproduction Problems, Irkutsk, the Russian Federation.E-mail: anait_24@mail.ru 
19. Chandra A, Surti N, Kesavan S, Agarwal A. Significance of oxidative stress in human reproduction. Arch Med Sci. 2009;5:S28-S42.

20. Kolesnikova LI, Darenskaya MA, Kolesnikov SI. [Free radical oxidation: a pathophysiologist's view]. Bulletin of Siberian Medicine. 2017;16(4):16-29. doi: 10.20538/16820363-2017-4-16-29. [Article in Russizn].

21. Kolesnikova L, Grebyonkina L, Darenskaya M, Vlasov BJ. Oxidative stress as a nonspecific pathogenic link of reproductive disorders (review). Bulletin of the Siberian Branch of the Russian Academy of Medical Sciences. 2012; 32(1): 58-66.

22. Marianian A, Protopopova N, Kolesnikova L. Metabolic changes in newborns and women who consumed small doses of alcohol in the prenatal period. Journal of Pharmaceutical Research International. 2020;32(24):1-8. doi: 10.9734/ JPRI/2020/v32i2430803

23. Kolesnikova LI, Darenskaya MA, Grebenkina LA, Labygina AV, Suturina LV, Dolgikh MI et al. Activity of lipid peroxidation in infertile women from different populations. Bull Exp Biol Med. 2012;154(2):203-205.

24. Lash LH. Mitochondrial glutathione transport: Physiological, pathological and toxicological implications. Chem Biol Interact. 2006;163(1-2):54-67. doi: 10.1016/j. cbi.2006.03.001.

25. Menshchikova E, Zenkov N, Lankin V, Bondar I, Trufankin V. Oxidative stress: Pathological conditions and diseases. Novosibirsk, Art.: 2008. [In Russian].

26. Niki E. Lipid peroxidation: Physiological levels and dual biological effects. Free Radic Biol Med. 2009;47(5):469-484. doi: 10.1016/j.freeradbiomed.2009.05.032

27. Balachova T, Bonner BL, Chaffin M, Isurina G, Shapkaitz V, Tsvetkova L, Volkova E, Grandilevskaya I, Skitnevskaya L, Knowlton N. Brief FASD prevention intervention: physicians' skills demonstrated in a clinical trial in Russia. Addict Sci Clin Pract. 2013 Jan 8;8(1):1. doi: 10.1186/1940-0640-8-1.

28. Balachova $\mathrm{T}$, Bonner $\mathrm{B}$, Isurina G. Preventing fasd in russia (8th ed, vol. 7). Fetal Alcohol Forum. The FASD Medical e-Network NOFAS-UK.: 2012.

29. Hannigan JH, Chiodo LM, Sokol RJ, Janisse J, Ager JW, Greenwald MK at al. A 14-year retrospective maternal report of alcohol consumption in pregnancy predicts pregnancy and teen outcomes. Alcohol. 2010;44(7-8):583-594. doi: 10.1016/j.alcohol.2009.03.003.

30. Alvik A, Haldorsen T, Groholt B, Lindemann R. Alcohol consumption before and during pregnancy comparing concurrent and retrospective reports. Alcohol Clin Exp Res. 2006;30(3):510-515. doi: 10.1111/j.1530-0277.2006.00055.x. 31. Babor TF, Steinberg K, Anton R, Del Boca F. Talk is cheap: Measuring drinking outcomes in clinical trials. J Stud Alcohol. 2000;61(1):55-63. doi: 10.15288/jsa.2000.61.55.
32. Maisto SA, Connors GJ. Using subject and collateral reports to measure alcohol consumption. Humana Press: 1992. 33. Sobell L, Sobell M. Alcohol consumption measures. Avaiable from: https://pubs.niaaa.nih.gov/publications/ assessingalcohol $/$ measures.htm

34. Kesmodel U, Kesmodel PS, Larsen A, Secher NJ. Use of alcohol and illicit drugs among pregnant danish women, 1998. Scand J Public Health 2003;31(1):5-11. doi: 10.1080/14034940210134202.

35. Kristjanson AF, Wilsnack SC, Zvartau E, Tsoy M, Novikov B. Alcohol use in pregnant and nonpregnant russian women. Alcohol Clin Exp Res. 2007;31(2):299-307. doi: 10.1111/j.1530-0277.2006.00315.x.

36. National Institute on Alcohol Abuse and Alcoholism (NIAAA). Helping patients who drink too much: A clinician's guide. Avaiable from: https://pubs.niaaa.nih.gov/publications/ practitioner/cliniciansguide2005/guide.pdf

37. Hissin PJ, Hilf R. A fluorometric method for determination of oxidized and reduced glutathione in tissues. Anal Biochem. 1976;74(1):214-226. doi: 10.1016/0003-2697(76)90326-2.

38. Taylor SL, Lamden MP, Tappel AL. Sensitive fluorometric method for tissue tocopherol analysis. Lipids. 1976;11(7):530538. doi: 10.1007/BF02532898.

39. Corongiu FP, Banni S. Detection of conjugated dienes by second derivative ultraviolet spectrophotometry. Methods Enzymol. 1994;233:303-310. doi: 10.1016/s00766879(94)33033-6.

40. Yagi K. Assay for serum lipid peroxide level and its clinical significance. Academic Press: 1982.

41. Townsend DM, Tew KD, Tapiero H. The importance of glutathione in human disease. Biomed Pharmacother. 2003;57(3-4):145-155. doi: 10.1016/s0753-3322(03)00043-x. 42. Franco R, Cidlowski JA. Apoptosis and glutathione: Beyond an antioxidant. Cell Death Differ. 2009;16:13031314. doi.org/10.1038/cdd.2009.107.

43. Marianian A, Atalyan A, Bohora S, Darenskaya M, Grebenkina L, Kolesnikova L at al. The effect of low alcohol consumption during pregnancy on the lipid peroxidationantioxidant defense system of women, their alcohol-exposed infants, and growth, health, and developmental outcomes. Journal of Birth Defects Research. 2020;112(1):40-53. doi: 10.1002/bdr2.1582.

44. Marianian AY, Kolesnikova LI, Protopopova NV, Kalinkina OB. Influence of small doses of alcohol on the state of health of pregnant women and their newborns. Alcoholism: Clinical and Experimental Research. 2018;42(6):77. doi: 10.1111/acer.13747.

45. Burina EA, Kulieva AK, Marianian AY. The risk of fetal alcohol syndrome in pregnant women and women planning pregnancy. Alcoholism: Clinical and Experimental Research. 2018;42;(6):75. doi: 10.1111/acer.13833. 